\title{
Nucleation and growth of supported clusters at defect sites: $\mathrm{Pd} / \mathrm{MgO}(001)$
}

\author{
G. Haas, A. Menck, H. Brune, and J. V. Barth \\ Institut de Physique Expérimentale, EPFL, CH-1015 Lausanne, Switzerland \\ J. A. Venables \\ Department of Physics and Astronomy, Arizona State University, Tempe, Arizona 85287 \\ and CPES, University of Sussex, Brighton BN1 9QH, United Kingdom \\ K. Kern \\ Institut de Physique Expérimentale, EPFL, CH-1015 Lausanne, Switzerland \\ and Max-Planck-Institut für Festkörperforschung, D-70569 Stuttgart, Germany
}

(Received 4 November 1999)

\begin{abstract}
Nucleation and growth of $\mathrm{Pd}$ on cleaved $\mathrm{MgO}(001)$ surfaces were studied by variable-temperature atomic force microscopy in the temperature range $200-800 \mathrm{~K}$. Constant island densities $\left(\sim 3 \times 10^{12} \mathrm{~cm}^{-2}\right)$ were observed over a wide temperature range, indicating nucleation kinetics governed by point defects with a high trapping energy. These results are compared to a rate equation model that describes the principal atomistic nucleation and growth processes, including nucleation at attractive point defects. Energies for defect trapping, adsorption, surface diffusion, and pair binding are deduced, and compared with recent $a b$ initio calculations.
\end{abstract}

Metal aggregates supported on oxide surfaces have many practical applications due to their catalytic, magnetic, and electrical properties. Consequently, fundamental studies have been carried out on a range of model systems. ${ }^{1}$ Although the main microscopic steps governing nucleation and growth of the films are now understood, detailed characterization of these processes has proven difficult. In particular, little is known about the energies involved. In recent years, $a b$ initio calculations of the binding of metal atoms and clusters to oxide surfaces have progressed, generating further stimulus for experimental determination of the relevant interactions. On the other hand, a much more complete understanding has been achieved for the case of metallic substrates. In large part, this is due to the application of variable-temperature scanning tunneling microscopy (STM) for in situ imaging of the nucleation and growth stages, compared to analytic models and numerical simulations. ${ }^{2}$

In this work, we adopt a similar approach to metal growth on insulating oxide surfaces. This allows us to determine the principal energies governing nucleation and growth, and in particular to consider the influence of defects. On oxides, defects like vacancies or steps are present even at wellprepared single-crystal surfaces. Frequently the nucleation and growth behavior on these substrates is dominated by the defects. Early transmission electron microscopy studies of metal growth on alkali halide and alkaline earth surfaces ${ }^{3}$ indicated defect nucleation in some cases, but relatively little is known about the interaction between defects and adsorbed metal atoms.

We have studied the growth of $\mathrm{Pd}$ on $\mathrm{MgO}(001)$ surfaces, utilizing variable-temperature atomic force microscopy (AFM) to image the deposits. This is one of the most explored metal/oxide systems, being a model for supported metal catalysts. Palladium grows in three-dimensional clusters, similar to most metal/oxide systems, as the surface energy is usually higher for metals than for oxides. ${ }^{1}$ We dem- onstrate that the nucleation kinetics in a large temperature range is determined by attractive point defects. With the help of a simple rate equation model we deduce the relevant interaction energies for the $\mathrm{Pd} / \mathrm{MgO}(001)$ system. It is expected that the understanding of nucleation at defects can be explored to produce novel nanostructures.

The experiments were performed with a variabletemperature atomic force microscope, mounted in a standard UHV chamber. The AFM, of the Besocke Beetle type, utilizes piezoresistive cantilevers ${ }^{4}$ for force detection. This setup allows easy tip exchange without breaking vacuum, and imaging of the $\mathrm{MgO}$ samples in the temperature range $100-500 \mathrm{~K}$ in contact and noncontact modes. Magnesium oxide disks $2.7 \mathrm{~mm}$ thick and $23 \mathrm{~mm}$ in diameter were prepared by cleaving a single-crystal $\operatorname{rod}^{5}$ along the (001) plane under Ar gas and introduced into UHV by a load-lock without exposing them to ambient atmosphere. Heating and cooling were achieved by thermal contact to the sample holder, which could be electron-beam heated and liquid-nitrogen or helium cooled. The sample temperature was calibrated by a $0.1 \mathrm{~mm}$ NiCr-Ni thermocouple pair glued to the center of a $\mathrm{MgO}$ sample before the experiments. Prior to deposition of $\mathrm{Pd}$ the crystal was heated in oxygen $\left(10^{-4}\right.$ mbar, $750 \mathrm{~K}, 30$ $\min )$.

Atomic force microscopy imaging of the surface revealed flat terraces $30-500 \mathrm{~nm}$ wide and typically several $\mu \mathrm{m}$ long, separated by mono- or multilayer steps. Palladium was deposited from an electron-beam-heated Pd rod evaporator (0.9 $\mathrm{kV}$ electrons, Omicron EFM-4) in a position vertical to the sample surface, with a voltage $(+1.25 \mathrm{kV})$ applied to an aperture between rod and sample to avoid ions reaching the surface. In prior cryotemperature Pd deposition experiments on $\operatorname{Pd}(110)$, the flux was calibrated against the ion current by counting the deposited atoms with a low-temperature STM. For the experiments presented here, a coverage of about 0.1 monolayer (ML) was deposited at a rate of 3.2 


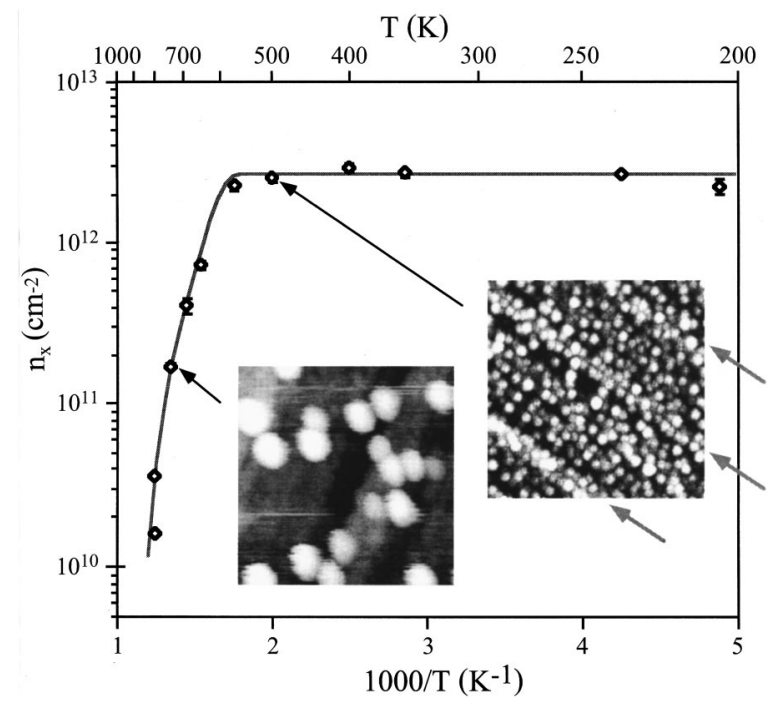

FIG. 1. Arrhenius representation of Pd island density $n_{x}\left(\mathrm{~cm}^{-2}\right)$ at $0.1 \mathrm{ML}$ coverage. The solid line is a "best fit" obtained with a rate equation model for $E_{d}=0.2, E_{t}=1.5, E_{b}=1.2$, and $E_{a}$ $=1.2 \mathrm{eV}$. Insets: noncontact AFM images of Pd deposits on Arcleaved $\mathrm{MgO}(001)$. The substrate temperature during deposition was 500 and $745 \mathrm{~K}$ for these two images. Three arrows point along steps in the $500 \mathrm{~K}$ micrograph. The size is $100 \times 100 \mathrm{~nm}^{2}$ for both images.

$\times 10^{-2} \mathrm{ML} / \min \left(1 \mathrm{ML}=1.13 \times 10^{15} \mathrm{~cm}^{-2}\right)$. Auger electron spectra (AES) recorded after deposition revealed no change in sticking of Pd between 200 and $750 \mathrm{~K}$, indicating complete condensation in that temperature range. On the other hand, condensation was clearly incomplete at $800 \mathrm{~K}$. With a nominal exposure to $0.1 \mathrm{ML}$ Pd no islands were seen by AFM and about ten times more Pd had to be evaporated onto the sample to get a similar Pd AES signal.

The influence of the deposition parameters, substrate temperature and flux, has been studied in detail. Atomic force microscopy images were obtained in situ after Pd deposition (see the insets in Fig. 1). We chose to image the particles in the noncontact mode, since in contact mode (forces down to $10 \mathrm{nN}$ ) the small Pd particles were displaced by the tip to the borders of the scanned region. For each experiment, Pd was evaporated at a different substrate temperature onto a freshly cleaved $\mathrm{MgO}$ surface. For deposition temperatures above $300 \mathrm{~K}$ the sample was quenched to room temperature before imaging. Below $300 \mathrm{~K}$, on the other hand, the AFM images were recorded at the deposition temperature to avoid changes in the island density. Since the AFM images represent a convolution of the island shape with the AFM tip, even for the low coverage deposited $(0.1 \mathrm{ML})$ the surface seems largely covered at the lower temperatures.

The island density has been determined from AFM micrographs for a wide range of substrate temperature $T$ and deposition flux $F$. The curve in Fig. 1 shows an Arrhenius representation of the island density $n_{x}$. The density stays constant over a remarkably wide span of deposition temperature, characteristic of nucleation at defects. The island density of the plateau is the number density of defects that act as traps for $\mathrm{Pd}\left(\cong 3 \times 10^{12} \mathrm{~cm}^{-2}\right)$. We are not able to identify the nature of these defects unambiguously for the moment, but the majority of the islands are not at steps, which can be readily observed by AFM. These additionally act as nucleation centers, as is seen for the three steps in the $500 \mathrm{~K}$ inset in Fig. 1 (arrows). The typical island depletion zone around the steps is apparent in this image. But most of the islands are situated between the steps on the terrace. Our preparation technique of cleaving under Ar clearly results in a reproducible density of such defects. Measurements performed by us with $\mathrm{MgO}$ samples cleaved in situ in UHV gave a similar density. We assign the nucleation sites to point defects, most likely surface vacancies.

The influence of Pd flux $F$ is as follows. In the plateau region $(450 \mathrm{~K})$ the island density was unchanged when the flux was varied over two decades. At high temperatures (745 $\mathrm{K})$ the dependence is very weak. In a double logarithmic plot of $n_{x}$ versus $F$ we find a slope of 0.07 , well below the expected values between $\frac{2}{7}$ and 1 for the case of homogeneous nucleation, depending on the size of the critical nucleus. ${ }^{7}$

There are other examples in the literature where nucleation on surface point defects takes place. ${ }^{3,8,9}$ In recent examples plateaus in the island density with temperature were found with $\mathrm{Fe}$ and $\mathrm{Cd}$ growth on $\mathrm{CaF}_{2}$ surfaces ${ }^{8}$ and $\mathrm{Fe}$ growth on UHV-cleaved $\mathrm{MgO}(001) .{ }^{9}$ In the latter work a constant island density of $6 \times 10^{12} \mathrm{~cm}^{-2}$, very comparable to the value observed in the present study, was found between 200 and $500 \mathrm{~K}$, which suggests the operation of a similar growth mechanism.

Defects can be incorporated into either analytical treatments or simulations, at the cost of at least two additional material parameters, the trap density $n_{t}$ and energy $E_{t}$. The maximum or saturation nucleation density has been derived ${ }^{8}$ by extending the equation for homogeneous nucleation. ${ }^{7}$ For nucleation at defects this equation is modified by a factor $\left(1+A_{t}\right)$, where $A_{t}$ is the ratio of the defect-induced to homogeneous nucleation rate.

Our reasoning can be understood using Fig. 2. Local equilibrium is quickly established between adatoms on terraces (density $\left.n_{1}\right)$ and at defect sites $\left(n_{1 t}\right)$. This yields a Langmuir adsorption isotherm for the occupation of traps, including the effect of clusters nucleated on traps $\left(n_{x t}\right)$ which block further adsorption. In the simplest case, where the traps act only on the first atom that joins them, and local entropic effects are ignored, we have

$$
n_{1 t} /\left(n_{t}-n_{x t}\right)=A /(1+A)
$$

where $A=n_{1} \exp \left(E_{t} / k T\right)$. Thus for $A \gg 1$, strong trapping, almost all the sites unoccupied by clusters are occupied by adatoms. In this model $A_{t}=n_{1 t} / n_{1}$; using these links betwen $A_{t}, A, n_{1}$ and $n_{x}$, the critical nucleus size $i$ and the regime of condensation (complete or incomplete) are determined selfconsistently as an output of an iterative calculation for given input values of adsorption, diffusion, and binding energies $\left(E_{a}, E_{d}, E_{b}\right)$ plus $E_{t}{ }^{8}$ Nucleation on terrace sites is strongly suppressed, due to adatom capture by clusters already nucleated on traps. However, when $n_{x}>n_{t}$, there is little effect on the overall nucleation density. This model results in the S-shaped curves shown in Fig. 3(a) for the whole temperature range studied, illustrated for $n_{t}=2.65 \times 10^{-3} \mathrm{ML}, E_{t}$ $=1.5 \mathrm{eV}, E_{b}$ and $E_{a}=1.2 \mathrm{eV}$, and $E_{d}$ in the range $0.2-0.4$ $\mathrm{eV}$, with an assumed value of the diffusion frequency factor 


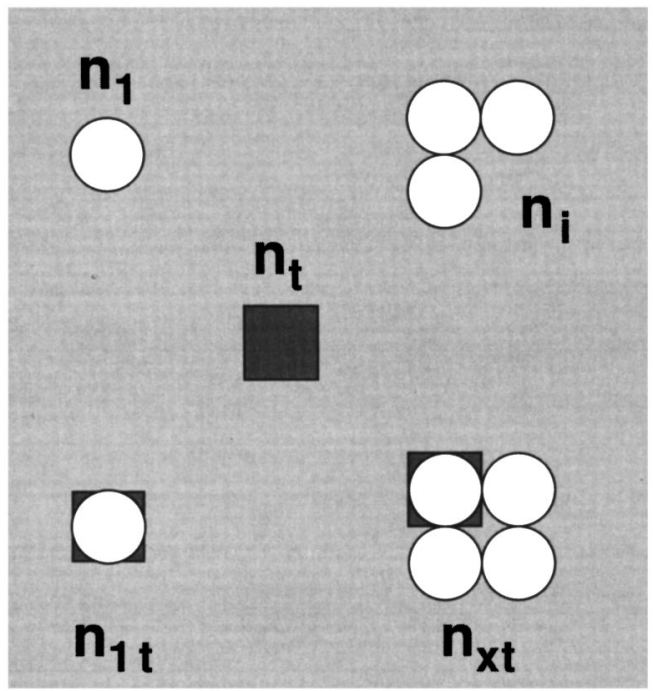

FIG. 2. Model for nucleation at randomly placed point defects with variables $n_{1}$, number density of free adatoms; $n_{t}$, density of traps; $n_{1 t}$, density of trapped adatoms; $n_{i}$, density of critical clusters (shown for the case of $i=3$, the critical cluster for the case $i$ $=1$ being a single adatom); $n_{x t}$, density of trapped stable clusters (clusters with atom numbers $>i$ ). The total density of stable clusters (trapped and free) is $n_{x}$. See text for discussion.

$\nu_{d}=3 \mathrm{THz}$, which is appropriate for bulk $\mathrm{Pd}$, if not for $\mathrm{Pd}-$ $\mathrm{MgO}$ where it may well be lower.

Comparison with the $\mathrm{Pd} / \mathrm{MgO}$ experiments allows us to deduce the following points: to reproduce the large extent in temperature of the plateau the trapping energy $E_{t}$ has to be high, $\geqslant 1.2 \mathrm{eV}$, and the diffusion energy must be low, $\leqslant 0.3$ $\mathrm{eV}$, as can be seen in Figs. 3(a) and 3(b). The reason a low value of $E_{d}$ is needed is so that the adatoms can migrate far enough at low temperatures to reach the defect sites. For weak trapping $(A<1)$, the main effect would be caused by the reduced diffusion constant $D$ due to the time adatoms spend at traps, yet this case is in disagreement with the extent of the plateau, and the rapid drop-off at higher temperatures. Thus, with such a high value of $E_{t}$, something else eventually intervenes at high temperatures.
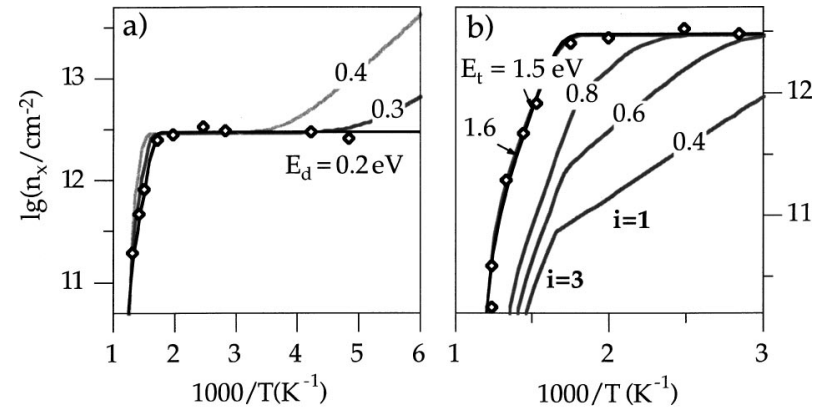

FIG. 3. (a) Nucleation density predicted with $n_{t}=2.65$ $\times 10^{-3} \mathrm{ML}, E_{t}=1.5 \mathrm{eV}, E_{a}=1.2 \mathrm{eV}$, and $E_{b}=1.2 \mathrm{eV}$, for various values of diffusion energy $E_{d}$ as indicated, close to the best fit. (b) Nucleation density predicted with trap density $n_{1}=2.65$ $\times 10^{-3} \mathrm{ML}, E_{a}$ and $E_{b}=1.2 \mathrm{eV}, E_{d}=0.2 \mathrm{eV}$, and various values of trap energy $E_{t}$, as indicated. The bend in the curves at about $1000 / T=1.7 \mathrm{~K}^{-1}$ is associated with the transition from a critical nucleus of $i=1$ to $i=3$. See text for further discussion.
There are two possibilities, given that an ad-dimer forms a stable nucleus at least up to $T \cong 600 \mathrm{~K}$. One possibility is that condensation becomes incomplete at this point, but that addimers remain stable, $i=1$. This would indicate a lower limit to the value of $E_{b}$, with a moderate value of $E_{a}$ being the important parameter. The other possibility is the inverse, where the first process to intervene is the transition to $i=3$ [due to the square (001) geometry], so that high-temperature data determine $E_{b}$, and only at even higher temperatures is the condensation incomplete. This means that the limiting process can become breakup of the cluster (on a trap), rather than removal of the adatom from the trap; $E_{t}$ is not then itself important, provided it is high enough. As condensation becomes incomplete only around $800 \mathrm{~K}$, the first scenario is clearly ruled out. Figure 3(b) shows that $i=1$ at low temperatures, but that the transition to $i=3$ is responsible for the initial drop-off at high temperatures, followed by incomplete condensation at the requisite temperature to agree with observations.

In the framework of the model this interpretation is unambiguous. If $E_{b}$ is increased markedly then the transition to $i=3$ is delayed to higher temperatures; $E_{a}$ then has to be reduced to fit the knee of the curve at $600 \mathrm{~K}$, but now the higher-temperature portion of the curve is much too steep, and incomplete condensation sets in too early. If $E_{b}$ is reduced below about $1.0 \mathrm{eV}$ the transition to $i=3$ occurs too readily to fit the knee of the curve at $600 \mathrm{~K}$, independent of the values of $E_{t}$ or $E_{a}$.

This plot is therefore close to our "best fit" added in Fig. 1 , indicating that $E_{a}$ and $E_{b}$ are $\cong 1.2 \mathrm{eV}$ for $\mathrm{Pd} / \mathrm{MgO}(001)$, $E_{d}<0.3 \mathrm{eV}$, and $E_{t}>1.2 \mathrm{eV}$. Our confidence in these values is about $0.2 \mathrm{eV}$, mainly because only an upper limit for $E_{d}$ has been determined, and its uncertainty is also reflected in the high-temperature features of the curve as apparent from Fig. 3(a).

There are two types of comparison that can be made with other work, notably with experiments by Henry and co-workers, ${ }^{6}$ and with $a b$ initio and other cluster calculations. ${ }^{10-15}$ Recent density-functional and other estimates of $E_{a}$ gave $0.9-1.0 \mathrm{eV},{ }^{10}$ with correction downward toward $0.8 \mathrm{eV} .^{10,11}$ One-quarter ML Pd was calculated to be bound to the surface by about $1.3 \mathrm{eV} /$ atom, ${ }^{12}$ encouraging us to believe that $E_{a}$ is of this order. There are several literature estimates of the binding energy of the diatomic molecule $\mathrm{Pd}_{2}$ in the gas phase, covering a huge range from 0.73 to 1.69 $\mathrm{eV}$. However, empirical work and some recent calculations prefer the lower end of the range, with all calculations below $1.35 \mathrm{eV} .{ }^{13}$ Given that values on surfaces are almost certainly lower than free-space values, $E_{b} \cong 1.2 \mathrm{eV}$ may well be quite reasonable. A rough evaluation of the diffusion barrier for the collective migration of a palladium monolayer on $\mathrm{MgO}$ has been given as $0.3 \mathrm{eV} .^{14}$

Finally, the trapping energy of $\mathrm{Pd}$ in a surface oxygen vacancy has been estimated to be as high as $2.55 \mathrm{eV},{ }^{15}$ provided the defect is a neutral $F_{s}$ center, which has two electrons located between the vacancy and the Pd adatom. The nature of trapping defects on $\mathrm{MgO}(001)$ is, however, not yet clear. Recent density-functional theory calculations of $\mathrm{Pt} /$ $\mathrm{MgO}(001)$ have suggested that monovacancies $\left(F_{s}\right.$ and $V_{s}$ centers) are strong traps only for the first $\mathrm{Pt}$ atom. ${ }^{16}$ In this calculation the next Pt adatom does not bond, i.e., the defect- 
trapped dimer is unstable. This led to the suggestion that $\mathrm{Mg}-\mathrm{O}$ divacancies are effective traps in generating stable nuclei. Clearly more theoretical work is needed to identify the nature of the trapping sites for $\mathrm{Pd} / \mathrm{MgO}(001)$ unambiguously.

The island densities and the values for $E_{a}$, and in particular for $\left(E_{a}-E_{d}\right)$, that we have deduced are considerably higher than those deduced from a series of experiments by Henry and co-workers. ${ }^{1,6}$ While we cannot at this stage uniquely identify all the causes of these differences, we note that if one has to invoke a spectrum of defect energies in order to explain a continuous variation of $n_{x}$ with temperature, then the model contains too many parameters (either explicit or implied) to achieve a unique answer. What is clear from the experiments reported here is that our sample preparation technique produces a high density of one type of defect, presumably a surface vacancy, which has a very high trapping energy for Pd adatoms. Under these circumstances the interpretation is clear, provided one remains within the simplest model that explains the results. It is encouraging that the energies needed within this model are close to those resulting from state-of-the-art cluster calculations.

The present study demonstrates that good understanding of the interaction of metals with oxide surfaces can be obtained by AFM experiments combined with a rate equation analysis. The observed trapping and nucleation of Pd clusters at attractive point defects are interesting from a fundamental point of view, but may also be of interest for producing novel supported nanostructures.
${ }^{1}$ C. T. Campbell, Surf. Sci. Rep. 27, 1 (1997); C. R. Henry, ibid. 31, 231 (1998).

${ }^{2}$ H. Brune, H. Röder, C. Baragno, and K. Kern, Phys. Rev. Lett. 73, 1955 (1994); H. Brune, Surf. Sci. Rep. 31, 121 (1998); see also Growth and Properties of Ultrathin Epitaxial Layers, The Chemical Physics of Solid Surfaces and Heterogeneous Catalysis Vol. 8, edited by D. A. King and D. P. Woodruff (Elsevier, Amsterdam, 1997), Chaps. 1, 5.

${ }^{3}$ M. Harsdorff, Thin Solid Films 90, 1 (1982); 116, 55 (1984); J. L. Robins, Appl. Surf. Sci. 33/34, 379 (1988). For a recent discussion of energies on alkali halides, see J. H. Harding, A. M. Stoneham, and J. A. Venables, Phys. Rev. B 57, 6715 (1998).

${ }^{4}$ Piezolevers ${ }^{\mathrm{TM}}$ from Park Scientific Instruments, U.S.A.; M. Tortonese, R. C. Barrett, and C. F. Quate, Appl. Phys. Lett. 62, 834 (1993).

${ }^{5}$ Crystal rods obtained from Kristallhandel Kelpin, Germany.

${ }^{6}$ C. Chapon, C. R. Henry, and A. Chenam, Surf. Sci. 162, 747 (1985); M. Meunier and C. R. Henry, ibid. 307-309, 514 (1994); C. R. Henry and M. Meunier, Vacuum 50, 157 (1998).

${ }^{7}$ J. A. Venables, Philos. Mag. 27, 697 (1973); Phys. Rev. B 36, 4153 (1987); for a review, see Surf. Sci. 299/300, 798 (1994) or Refs. 2.

${ }^{8}$ K. R. Heim, S. T. Coyle, G. G. Hembree, J. A. Venables, and M. R. Scheinfein, J. Appl. Phys. 80, 1161 (1996); for reviews of defect nucleation models, see J. A. Venables, in Structure and
Evolution of Surfaces, edited by R. C. Cammarata, E. H. Chason, T. L. Einstein, and E. D. Williams, MRS Symposia Proceedings No. 440 (Materials Research Society, Pittsburgh, 1997), p. 129; Physica A 239, 35 (1997).

${ }^{9}$ G. Fahsold, A. Pucci, and K. H. Rieder, Phys. Rev. B 61, 8475 (2000).

${ }^{10}$ I. V. Yudanov, S. Vent, G. Pacchioni, K. Neyman, and N. Rösch, Chem. Phys. Lett. 275, 245 (1997); K. M. Neyman, S. Vent, G. Pacchioni, and N. Rösch, Nuovo Cimento D 19, 1743 (1997); N. López and F. Illas, J. Phys. Chem. B 102, 1430 (1998).

${ }^{11}$ A. Stirling, I. Gunji, A. Endou, Y. Oumi, M. Kubo, and A. Miyamoto, J. Chem. Soc., Faraday Trans. 93, 1175 (1997).

${ }^{12}$ J. Goniakowski, Phys. Rev. B 58, 1189 (1998).

${ }^{13}$ I. Shim and K. A. Gingerich, J. Chem. Phys. 80, 5107 (1984); M. D. Morse, Chem. Rev. 86, 1049 (1986); K. Balasubramanian, J. Chem. Phys. 89, 6310 (1988); M. Harada and H. Dexpert, J. Phys. Chem. 100, 565 (1996); G. Valerio and H. Toulhoat, ibid. 100, 10827 (1996); J. M. Seminario, A. G. Zacarías, and M. Castro, Int. J. Quantum Chem. 61, 515 (1997). We acknowledge J. H. Harding for useful correspondence.

${ }^{14}$ J. Goniakowski, Phys. Rev. B 57, 1935 (1998).

${ }^{15}$ A. M. Ferrari and G. Pacchioni, J. Phys. Chem. 100, 9032 (1996); A. V. Matveev, K. M. Neyman, I. V. Yudanov, and N. Rösch, Surf. Sci. 426, 123 (1999).

${ }^{16}$ A. Bogicevic and D. R. Jennison, Surf. Sci. 437, L741 (1999). 